\title{
Large-Scale Structure Probes of Modified Gravity
}

\author{
Catherine Heymans ${ }^{1}$ and Gong-Bo Zhao ${ }^{2}$ \\ ${ }^{1}$ Institute for Astronomy, University of Edinburgh, Royal Observatory, Blackford Hill, \\ Edinburgh, EH9 3 HJ. \\ heymans@roe.ac.uk \\ ${ }^{2}$ National Astronomy Observatories, Chinese Academy of Science, Beijing, 100012, P.R.China \\ ES \\ Institute of Cosmology \& Gravitation, University of Portsmouth, Dennis Sciama Building, \\ Portsmouth, PO1 3FX, UK \\ gbzhao@nao.cas.cn
}

\begin{abstract}
Observations of the evolution of large-scale structures in the Universe provides unique tools to confront Einstein's theory of General Relativity on cosmological scales. We review weak gravitational lensing and galaxy clustering studies, discussing how these can be used in combination in order to constrain a range of different modified gravity theories. We argue that in order to maximise the future information gain from these probes, theoretical effort will be required in order to model the impact of beyond-Einstein gravity in the non-linear regime of structure formation.

Keywords: Observational Cosmology; Gravitational Lensing; Galaxy Clustering; Surveys

PACS numbers:
\end{abstract}

\section{Introduction}

There is a multitude of potentially viable beyond-GR theories that introduce a variety of new scalar-field degrees of freedom. These new fields can couple to the matter fields in the Universe, modifying their gravitational interactions through a 'fifth force'. How these beyond-GR theories differ from Einstein's famed cosmological constant could be considered somewhat ambiguous, as in principle a 'cosmological constant' can be placed on either side of Einstein's field equations to modify them. With what we'll term 'modified gravity' theories, the modification is made to the 'curvature' side of the equations, the source of the gravitational field. With what we'll term 'dark energy' theories, uncoupled scalar fields, are seen as a new energy component modifying the 'stress-energy' tensor. Modified gravity theories, dark energy theories and the cosmological constant, are therefore all modifications of GR. Where the difference between these theories is unambiguous however is how they impact the expansion history of the Universe and the growth of structures over time ${ }^{1}$ Only the theories that couple to the matter field will contribute scaledependent alterations to the linear growth of density fluctuations, as illustrated in Figure 1. As such the observed growth of large-scale structures over time will be 
inconsistent with the growth predicted from the Hubble expansion alone. In order to confront a range of modified gravity theories and to separate them from dark energy theories, one therefore needs a combination of observations to probe both the expansion history and the growth of structures. In addition, in order to distinguish between modified gravity scenarios where theories can be broadly classed by their predictions for how matter bends space and time, and how the gravitational constant $G$ evolves, we also need observations that can test the 'curvature of space' (the curvature potential $\Phi$ ) independently from the 'curvature of time' (the Newtonian potential $\Psi)$.
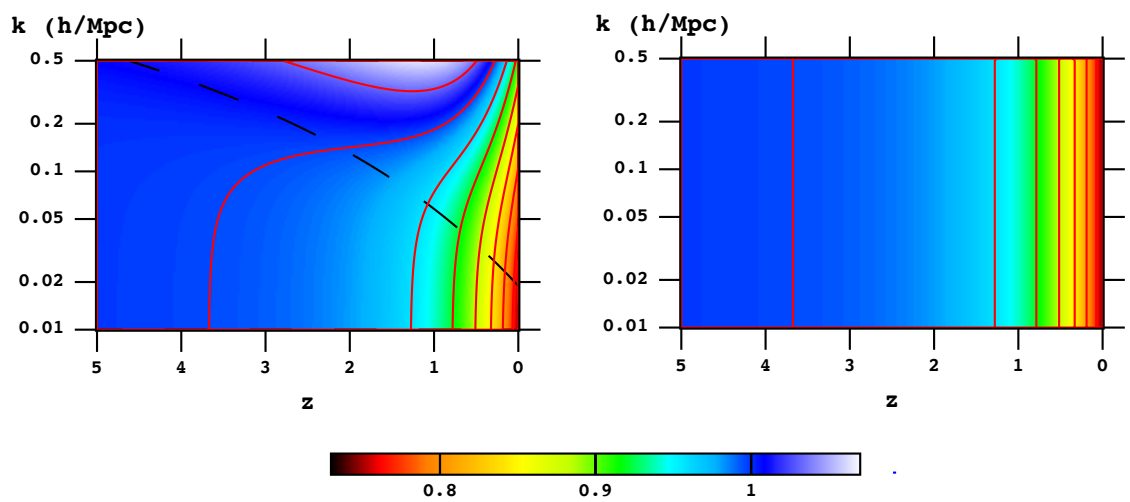

Fig. 1. The evolution of the growth factor for the linear matter power spectrum $\Delta_{m}(k, z)$, showing $\left[(1+z) \Delta_{m}(k, z)\right] /\left[6 \Delta_{m}(k, z=5)\right]$ as a function of redshift $z$ and scale $k$. The left panel corresponds to the Hu-Sawicki $f(R)$ mode ${ }^{2}$ where the $R+\Lambda$ term in the action of GR is replaced by a function of the Ricci scalar, $f(R)$. The promotion of $R$ to $f(R)$ introduces an additional degree of freedom making the growth of structures scale-dependent in comparison to the scale-independence of the $\Lambda \mathrm{CDM}$ case shown in the right panel. For this analysis the $f(R)$ function is set so that at the background level, $f(R) \simeq R+\Lambda$, with an effective dark energy equation of state $w_{\text {eff }}=-1$ and an amplitude $\left|f_{R 0}\right|=10^{-4}$. Figure reproduced from Ref $!^{3}$

Over the coming decade a number of large-scale cosmological surveys are coming online. The imaging surveys, the Large Synoptic Survey Telescope (LSST) and Euclid, will survey over 10,000 square degrees, mapping the evolution in the growth of large-scale structures of dark matter using a technique called weak gravitational lensing. The spectroscopic surveys, the Dark Energy Spectroscopic Instrument (DESI) and Euclid, will survey similar areas, mapping the 3-D distribution of galaxies across cosmic-time. These facilities used in combination with high-precision measurements of the early-Universe from the exquisite Planck observations of the cosmic microwave background (CMB) will provide a wealth of observational evidence to confront a range of different theories. In this chapter we describe three large-scale structure probes; baryon acoustic oscillations, redshift-space distortions and weak gravitational lensing, highlighting how, when used in combination, these 
probes can break degeneracies in constraining a range of beyond-GR theories.

\section{Large-Scale Structure probes}

\subsection{Gravitational Lensing}

Large-scale structures of dark matter gravitationally deflect light rays as they travel through the Universe, coherently distorting the distant galaxy images that we observe. This is often referred to as 'cosmic shear' (for a review see Ref. 4). Photons of light from different galaxies can act as relativistic particles for a large-scale cosmological gravity experiment. In its cosmological trajectory, the light travels through as much time as space, and hence, lensing is sensitive to the curvature of both space and time. As lensing probes both the universal expansion of the Universe and structure formation it is a powerful tool for the study of both standard and beyond-GR cosmological models.

Figure 2 shows the expected signal-to-noise of a measurement of the cosmic shear power spectrum $P_{\kappa}$ for a future deep, wide-area 15,000 square degree survey like the Large-Survey Synoptic Telescope (LSST ${ }^{5}$ ). $P_{\kappa}$ is the power spectrum of the projected surface mass density along the line of sight and is related to the matter power spectrum $P_{\delta}$ through

$$
P_{\kappa}(\ell)=\int_{0}^{\chi_{\mathrm{H}}} Q(\chi) P_{\delta}(\ell / \chi, \chi)
$$

where $\chi$ is the comoving radial distance and $Q(\chi)$ is a redshift-weight that combines information about the depth of the weak lensing survey with the lensing efficiency of foreground structures $\frac{4}{4}$ As $Q(\chi)$ peaks at very roughly half the redshift of the lensed background galaxy sample, for this $z>1$ LSST forecast from Ref. 6], we can see that the lensing measurement has its strongest constraining power in the deeply non-linear regime around physical scales of $k \sim 1 \mathrm{~h} \mathrm{Mpc}^{-1}$.

In the lower panel of Figure 2 we compare the cosmic shear power spectrum from Ref. 7 for two $f(R)$ gravity model $s^{2}$ and an example Dilaton model ${ }^{[8}$ Here the non-linear behaviour of the non-GR models has been calculated using a phenomenological halo model approach ${ }^{9}$ We can see that the most significant deviations from GR for these models are in the non-linear regime, where the weak lensing observations are very sensitive. This therefore strongly motivates the theoretical study of modifications to gravity in the non-linear regime. but with a strong caveat. Both observational systematics and the uncertain impact of baryon feedback on the dark matter non-linear matter power spectrum are likely to be significant on these scales $10[11$

\subsection{Baryon Acoustic Oscillations and Redshift Space Distortions}

Observationally, scale-dependent structure growth is a smoking gun of modified gravity, and it can in principle be probed by large spectroscopic galaxy surveys that map the Universe from the distant past to the present epoch. By measuring 


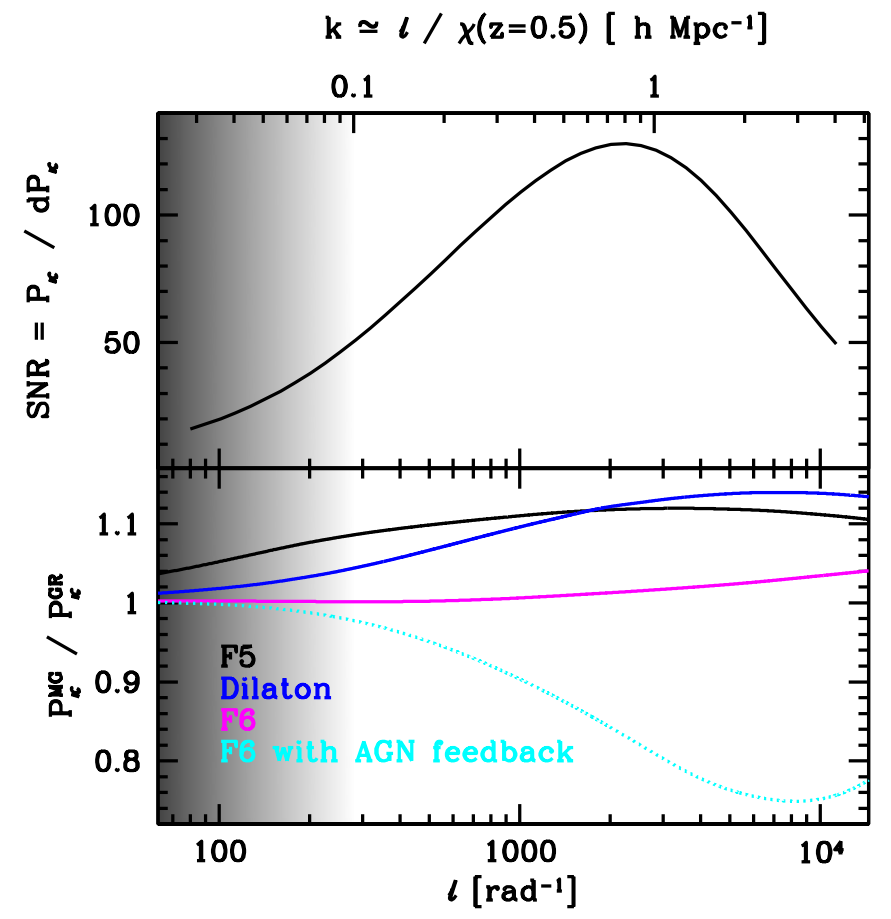

Fig. 2. Weak lensing is most sensitive to structures in the non-linear regime. In the upper panel, the forecasted signal-to-noise ratio for the shear power spectrum $P_{\kappa}(\ell)$ measured from a $z>1$ sample of galaxies in an LSST-like survey. The upper axis illustrates the physical $k$-scales which the projected weak lensing signal is most sensitive to. The shaded box indicates the transition from a linear (black) to a non-linear (white) regime. The lower panel shows the difference between the modified gravity and GR weak lensing power spectrum for $f(R)$ gravity with $\left|f_{R 0}\right|=10^{-5}$ (F5 - black), and with $\left|f_{R 0}\right|=10^{-6}$ (F6 - pink), and an example Dilaton model (blue). Baryon feedback also impacts the non-linear power spectrum on these scales. The dotted line compares a dark-matter only GR power spectrum with an F6 cosmology that also includes strong AGN feedback. The data used to create this figure was taken from Refs $6[7$

redshifts from spectra of a large number of galaxies, redshift surveys produce threedimensional maps of the Universe in redshift space. The statistical quantities of the clustering of the galaxies can then be used to confront a range of cosmological models.

Galaxy clustering measurements are often separated into two regimes. Largescale real-space clustering measurements are used to detect an enhanced galaxy clustering signal at $\sim 150 \mathrm{~h}^{-1} \mathrm{Mpc}$ that was imprinted in the baryonic matter distribution as a result of photon-baryon interaction in the early Universe. This fixed 'baryon acoustic oscillation' length scale in the distribution of galaxies acts as a standard ruler with which to probe the geometry and background expansion of the Universe ${ }^{12}$ Redshift-space clustering measurements look at the relative distortion in the clustering signal along and perpendicular to the line-of-sight that arises from the peculiar motions of galaxies ${ }^{[13}$ These 'redshift space distortions' (RSD) 
are driven on all scales by local gravitational potentials which means that these observations are directly related to gravity on cosmological scales and thus are a powerful probe of gravity models. There is however a caveat that the galaxies are a biased tracer of the total matter distribution and this bias needs to be accounted for when comparing observations to theoretical models.

\section{Observational constraints on modified gravity from combined probe analyses}

Individually both RSD and weak lensing observations can provide constraints on beyond-GR models of gravity (see for example a recent RSD analysis in Ref. 14), but their true power comes in their combination $\frac{15}{15}$. One approach taken by many so far is to look for consistency with GR in these observations by determining joint constraints on phenomenological modified gravity models to observations of RSD and weak lensing, in combination with geometry probes such as BAO and the cosmic microwave background (CMB).

One example of a phenomenological modified gravity model is to alter the Newtonian potential $\Psi$ and curvature potential $\Phi$ in the Friedmann-Robertson-Walker metric with a spatially scale-independent modification that scales with time in proportion to the effective dark energy density $15|17| 19 \mid 21$;

$$
\begin{gathered}
\Psi_{\mathrm{MG}}(k, a)=\left[1+\mu_{0} \eta(a)\right] \Psi_{\mathrm{GR}}(k, a), \\
\Psi_{\mathrm{MG}}(k, a)+\Phi_{\mathrm{MG}}(k, a)=\left[1+\Sigma_{0} \eta(a)\right]\left[\Psi_{\mathrm{MG}}(k, a)+\Phi_{\mathrm{GR}}(k, a)\right],
\end{gathered}
$$

where $\eta(a)=\Omega_{\Lambda}(a) / \Omega_{\Lambda}(a=1)$. For the case of GR, $\Sigma_{0}=\mu_{0}=0$. Figure 3 shows current constraints for this MG parameterisation from Ref. 17 (panel C) and Ref. 18 (panel D). Both show constraints using weak lensing measurements from the Canada-France-Hawaii Telescope Lensing survey (CFHTLenS, ${ }^{[2]}$ panel A showing the tomographic shear correlation function $\left.\xi_{ \pm}(\theta)\right)$ and a compilation of redshift space distortion measurements (panel B showing $f \sigma_{8}$, where $f$ and $\sigma_{8}$ are the logarithmic growth rate and the root mean square of the density fluctuation on a scale of $8 \mathrm{Mpc} / \mathrm{h}$, respectively, see Ref[16 for details). Focussing on panel C we see that RSD (shown in green) is only sensitive to changes in the curvature of time $\mu_{0}$, as galaxies are non-relativistic tracers of the gravitational potential. Weak lensing (shown in red), however is sensitive to changes in both of the Bardeen potentials, as lensing is a relativistic tracer. The combination of these two probes breaks the degeneracy between these two phenomenological MG parameters and when combined with $\mathrm{BAO}^{23}$ and WMAP CMB observations ${ }^{24}$ there is good consistency found with GR. Panel D revisits this analysis using updated CMB constraints from Planck ${ }^{\sqrt{18}}$ finding some tension with the GR-prediction. This tension however stems from differences between the probes even in a standard flat $\Lambda$ CDM analysis. ${ }^{25}$ When combining Planck with weak lensing data from the Dark Energy Survey $26[27$ the results are found to be GR-consistent with $\mu_{0}=-0.07_{-0.32}^{+0.19}$ and $\Sigma_{0}=0.018_{-0.048}^{+0.059}$. As RSD and 

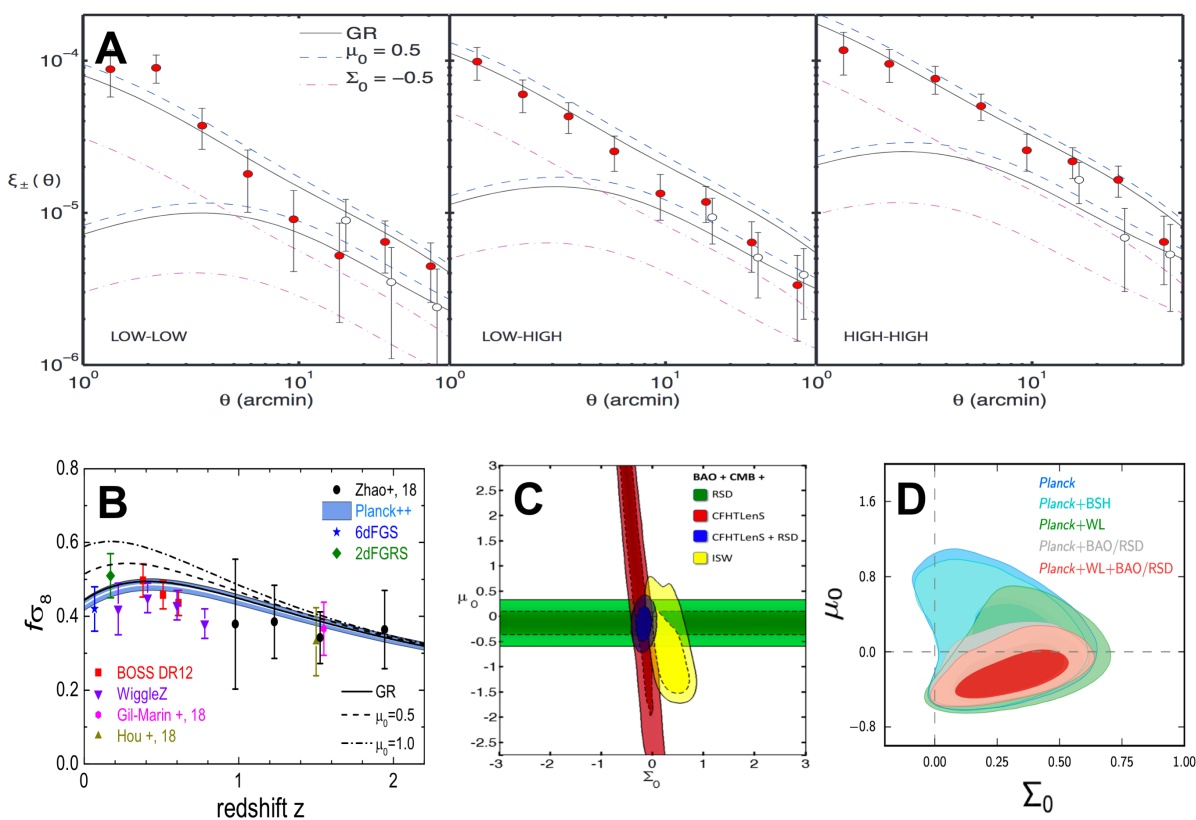

Fig. 3. A compilation of observational constraints. The impact of varying the parameterised modified gravity parameters $\mu_{0}$ and $\Sigma_{0}$ can be seen for weak gravitational lensing observables in panel A. Here the tomographic shear correlation function $\xi_{+}(\theta)$, closed circles, and $\xi_{-}(\theta)$, open circles, has been measured from the CFHT Lensing Survey (CFHTLenS) within a low (left) and high (right) redshift bin. The shear correlation between the two redshift bins is also shown (centre panel). A compilation of redshift space distortion measurements is shown in panel B, compared to two different $\mu_{0}$ values. RSD is insensitive to changes in $\Sigma_{0}$. For the details of the different surveys shown in panel B see Ref 16 Joint constraints on $\Sigma_{0}$ and $\mu_{0}$ are shown adopting CMB measurements from WMAP (panel C) and Planck (panel D, where BSH in the inset refers to the analysis that includes constraints from BAO, Supernovae and a prior on the Hubble constant). Figures reproduced from Refs 16 18

weak lensing observations improve in both precision and accuracy over the coming decade it will be interesting to see whether consistency or tension arises between these different and independent probes.

The 'gravitational slip' statistic, $E_{G} \frac{28 \mid 29}{20}$ provides an alternative statistic to discriminate between different MG theories by taking ratios of weak lensing, clustering and RSD measurements. Whilst a promising probe for modifications to gravity, Ref. 30 argue that as $E_{G}$ primarily constrains $\Omega_{m}$, all systematic sources of tension between the CMB and large-scale structure probes need to be ruled out before $E_{G}$ can be used as a reliable consistency test of GR. In addition Ref. 31 and 32 show that whilst the approach of taking ratios of observables reduces the impact of observational uncertainties relating to galaxy bias, it is still a significant source of error, 
reducing the arguably already limited constraining power of this statistic. The future focus is therefore on joint cosmological parameter inference over all large-scale structure and CMB observables.

A range of different phenomenological MG parameterisations exist $\sqrt{33} \sqrt[36]{36}$ in addition to well tested software made publicly available in order to to carry out these analyses $37 \sqrt[38]{38}$ It is however challenging to map any phenomenological constraints to the underlying theories that we wish to confront. Development in this area has been led by Refs 39, 40 that link the $\mu, \Sigma$ parameterisation from equations 2 and 3 . to general Horndeski theories, finding a series of consistency conditions that can discriminate against broad classes of theories. There has also been recent focus to use observations to directly constrain the parameters for Horndeski gravity models. $\frac{41,43}{43}$ All these developments are however, limited to the linear regime, where large-scale structure observations are least constraining (see Figure 22.

Observational constraints have so far primarily focused on analysing the twopoint statistics of the density field, but there is strong motivation to look to higherorder statistics $44 \sqrt[45]{4 n c l u d i n g}$ density-weighted two-points statistics $\frac{46}{48}$ We have argued the importance of analysing departures from GR in the non-linear regime. It therefore naturally follows that as this is also the regime where the density field becomes non-Gaussian, information will be lost by only analysing two-point statistics. Higher-order statistics can be challenging to model and measure observationally and can also be subject to a different set of systematics, ${ }^{49}$ but encouraging early result $\$ 50$ promotes the future importance of higher-order statistics to enhance modified gravity constraints.

\section{Conclusions}

Over the next decade we will see the results of the upcoming suite of 'Stage-IV' experiments that have been designed to constrain the dark energy equation of state parameter to the percent level. DESI has been designed so its RSD measurements will be able to distinguish between a range of MG theories at a high significance, and in combination with improved CMB observations from the Simon's Observatory, and weak lensing measurements from Euclid and LSST, a range of sophisticated GR tests can and will be performed on cosmological scales. Harnessing the full power of large-scale structure probes of gravity will, however, require significant theoretical developments to in order to accurately model modifications to gravity into the nonlinear regime.

\section{Acknowledgments}

We thank Dragan Huterer and Joachim Harnois-Deraps for kindly providing the data used to create Figure 2 $\mathrm{CH}$ and GBZ are supported by the European Research Council (ERC) under the European Union's Horizon 2020 research and innovation programme, grant agreement numbers 647112 "GLOBE" (CH) and 646702 "CosTesGrav" (GBZ). GBZ also acknowledges support from NSFC Grants 11720101004, 
11673025 and 11711530207, the National Basic Research Program of China (973

Program) (2015CB857004), and a Royal Society Newton Advanced Fellowship.

\section{References}

\section{References}

1. A. Joyce, L. Lombriser and F. Schmidt, Annual Review of Nuclear and Particle Science 66 (October 2016) 95, arXiv:1601.06133.

2. W. Hu and I. Sawicki, Phys. Rev. D 76 (November 2007) 104043, arXiv:0708.1190.

3. L. Pogosian and A. Silvestri, Phys. Rev. D 77 (January 2008) 023503, arXiv:0709.0296

4. M. Kilbinger, Reports on Progress in Physics 78 (July 2015) 086901, arXiv: 1411.0115

5. LSST Science Collaboration, P. A. Abell, J. Allison, S. F. Anderson, J. R. Andrew, J. R. P. Angel, L. Armus, D. Arnett, S. J. Asztalos, T. S. Axelrod and et al., ArXiv e-prints (December 2009) arXiv:0912.0201 [astro-ph.IM].

6. D. Huterer and D. L. Shafer, Reports on Progress in Physics 81 (January 2018) 016901, arXiv:1709.01091.

7. J. Harnois-Déraps, D. Munshi, P. Valageas, L. van Waerbeke, P. Brax, P. Coles and L. Rizzo, MNRAS 454 (December 2015) 2722, arXiv:1506.06313.

8. P. Brax, C. van de Bruck, A.-C. Davis and D. Shaw, Phys. Rev. D 82 (September 2010) 063519, arXiv:1005.3735

9. P. Brax and P. Valageas, Phys. Rev. D 88 (July 2013) 023527, arXiv:1305.5647.

10. E. Semboloni, H. Hoekstra, J. Schaye, M. P. van Daalen and I. G. McCarthy, MNRAS 417 (November 2011) 2020, arXiv:1105.1075.

11. R. Mandelbaum, ArXiv e-prints (October 2017) arXiv:1710.03235.

12. D. J. Eisenstein, I. Zehavi, D. W. Hogg, R. Scoccimarro, M. R. Blanton, R. C. Nichol, R. Scranton, H.-J. Seo, M. Tegmark, Z. Zheng, S. F. Anderson, J. Annis, N. Bahcall, J. Brinkmann, S. Burles, F. J. Castander, A. Connolly, I. Csabai, M. Doi, M. Fukugita, J. A. Frieman, K. Glazebrook, J. E. Gunn, J. S. Hendry, G. Hennessy, Z. Ivezić, S. Kent, G. R. Knapp, H. Lin, Y.-S. Loh, R. H. Lupton, B. Margon, T. A. McKay, A. Meiksin, J. A. Munn, A. Pope, M. W. Richmond, D. Schlegel, D. P. Schneider, K. Shimasaku, C. Stoughton, M. A. Strauss, M. SubbaRao, A. S. Szalay, I. Szapudi, D. L. Tucker, B. Yanny and D. G. York, ApJ 633 (November 2005) 560, astro-ph/0501171.

13. J. A. Peacock, S. Cole, P. Norberg, C. M. Baugh, J. Bland-Hawthorn, T. Bridges, R. D. Cannon, M. Colless, C. Collins, W. Couch, G. Dalton, K. Deeley, R. De Propris, S. P. Driver, G. Efstathiou, R. S. Ellis, C. S. Frenk, K. Glazebrook, C. Jackson, O. Lahav, I. Lewis, S. Lumsden, S. Maddox, W. J. Percival, B. A. Peterson, I. Price, W. Sutherland and K. Taylor, Nature 410 (March 2001) 169, astro-ph/0103143

14. E.-M. Mueller, W. Percival, E. Linder, S. Alam, G.-B. Zhao, A. G. Sánchez, F. Beutler and J. Brinkmann, MNRAS 475 (April 2018) 2122.

15. Y.-S. Song, G.-B. Zhao, D. Bacon, K. Koyama, R. C. Nichol and L. Pogosian, Phys. Rev. D 84 (October 2011) 083523, arXiv:1011.2106 [astro-ph.C0].

16. G.-B. Zhao, Y. Wang, S. Saito, H. Gil-Marín, W. J. Percival, D. Wang, C.-H. Chuang, R. Ruggeri, E.-M. Mueller, F. Zhu, A. J. Ross, R. Tojeiro, I. Pâris, A. D. Myers, J. L. Tinker, E. Burtin, P. Zarrouk, F. Beutler, F. Baumgarten, J. E. Bautista, J. R. Brownstein, K. S. Dawson, J. Hou, A. de la Macorra, G. Rossi, J. A. Peacock, A. G. Sánchez, A. Shafieloo, D. P. Schneider and C. Zhao, ArXiv e-prints (January 2018) arXiv: 1801.03043 . 
17. F. Simpson, C. Heymans, D. Parkinson, C. Blake, M. Kilbinger, J. Benjamin, T. Erben, H. Hildebrandt, H. Hoekstra, T. D. Kitching, Y. Mellier, L. Miller, L. Van Waerbeke, J. Coupon, L. Fu, J. Harnois-Déraps, M. J. Hudson, K. Kuijken, B. Rowe, T. Schrabback, E. Semboloni, S. Vafaei and M. Velander, MNRAS 429 (March 2013) 2249, arXiv: 1212.3339.

18. Planck Collaboration, P. A. R. Ade, N. Aghanim, M. Arnaud, M. Ashdown, J. Aumont, C. Baccigalupi, A. J. Banday, R. B. Barreiro, N. Bartolo and et al., A $₫ A \mathbf{5 9 4}$ (September 2016) A14, arXiv:1502.01590

19. L. Amendola, M. Kunz and D. Sapone, jcap 4 (April 2008) 013, arXiv:0704.2421

20. G.-B. Zhao, T. Giannantonio, L. Pogosian, A. Silvestri, D. J. Bacon, K. Koyama, R. C. Nichol and Y.-S. Song, Phys. Rev. D 81 (May 2010) 103510, arXiv:1003.0001 [astro-ph.CO].

21. C. D. Leonard, T. Baker and P. G. Ferreira, Phys. Rev. D 91 (April 2015) 083504, arXiv:1501.03509.

22. C. Heymans, L. Van Waerbeke, L. Miller, T. Erben, H. Hildebrandt, H. Hoekstra, T. D. Kitching, Y. Mellier, P. Simon, C. Bonnett, J. Coupon, L. Fu, J. Harnois Déraps, M. J. Hudson, M. Kilbinger, K. Kuijken, B. Rowe, T. Schrabback, E. Semboloni, E. van Uitert, S. Vafaei and M. Velander, MNRAS 427 (November 2012) 146, arXiv: 1210.0032

23. L. Anderson, E. Aubourg, S. Bailey, D. Bizyaev, M. Blanton, A. S. Bolton, J. Brinkmann, J. R. Brownstein, A. Burden, A. J. Cuesta, L. A. N. da Costa, K. S. Dawson, R. de Putter, D. J. Eisenstein, J. E. Gunn, H. Guo, J.-C. Hamilton, P. Harding, S. Ho, K. Honscheid, E. Kazin, D. Kirkby, J.-P. Kneib, A. Labatie, C. Loomis, R. H. Lupton, E. Malanushenko, V. Malanushenko, R. Mandelbaum, M. Manera, C. Maraston, C. K. McBride, K. T. Mehta, O. Mena, F. Montesano, D. Muna, R. C. Nichol, S. E. Nuza, M. D. Olmstead, D. Oravetz, N. Padmanabhan, N. PalanqueDelabrouille, K. Pan, J. Parejko, I. Pâris, W. J. Percival, P. Petitjean, F. Prada, B. Reid, N. A. Roe, A. J. Ross, N. P. Ross, L. Samushia, A. G. Sánchez, D. J. Schlegel, D. P. Schneider, C. G. Scóccola, H.-J. Seo, E. S. Sheldon, A. Simmons, R. A. Skibba, M. A. Strauss, M. E. C. Swanson, D. Thomas, J. L. Tinker, R. Tojeiro, M. V. Magaña, L. Verde, C. Wagner, D. A. Wake, B. A. Weaver, D. H. Weinberg, M. White, X. Xu, C. Yèche, I. Zehavi and G.-B. Zhao, MNRAS 427 (December 2012) 3435, arXiv:1203.6594

24. D. Larson, J. Dunkley, G. Hinshaw, E. Komatsu, M. R. Nolta, C. L. Bennett, B. Gold, M. Halpern, R. S. Hill, N. Jarosik, A. Kogut, M. Limon, S. S. Meyer, N. Odegard, L. Page, K. M. Smith, D. N. Spergel, G. S. Tucker, J. L. Weiland, E. Wollack and E. L. Wright, ApJS 192 (February 2011) 16, arXiv:1001.4635 [astro-ph.CO]

25. S. Joudaki, C. Blake, C. Heymans, A. Choi, J. Harnois-Deraps, H. Hildebrandt, B. Joachimi, A. Johnson, A. Mead, D. Parkinson, M. Viola and L. van Waerbeke, MNRAS 465 (February 2017) 2033, arXiv:1601.05786

26. M. A. Troxel, N. MacCrann, J. Zuntz, T. F. Eifler, E. Krause, S. Dodelson, D. Gruen, J. Blazek, O. Friedrich, S. Samuroff, J. Prat, L. F. Secco, C. Davis, A. Ferté, J. DeRose, A. Alarcon, A. Amara, E. Baxter, M. R. Becker, G. M. Bernstein, S. L. Bridle, R. Cawthon, C. Chang, A. Choi, J. De Vicente, A. Drlica-Wagner, J. Elvin-Poole, J. Frieman, M. Gatti, W. G. Hartley, K. Honscheid, B. Hoyle, E. M. Huff, D. Huterer, B. Jain, M. Jarvis, T. Kacprzak, D. Kirk, N. Kokron, C. Krawiec, O. Lahav, A. R. Liddle, J. Peacock, M. M. Rau, A. Refregier, R. P. Rollins, E. Rozo, E. S. Rykoff, C. Sánchez, I. Sevilla-Noarbe, E. Sheldon, A. Stebbins, T. N. Varga, P. Vielzeuf, M. Wang, R. H. Wechsler, B. Yanny, T. M. C. Abbott, F. B. Abdalla, S. Allam, J. Annis, K. Bechtol, A. Benoit-Lévy, E. Bertin, D. Brooks, E. Buckley-Geer, D. L. 
Burke, A. Carnero Rosell, M. Carrasco Kind, J. Carretero, F. J. Castander, M. Crocce, C. E. Cunha, C. B. D'Andrea, L. N. da Costa, D. L. DePoy, S. Desai, H. T. Diehl, J. P. Dietrich, P. Doel, E. Fernandez, B. Flaugher, P. Fosalba, J. García-Bellido, E. Gaztanaga, D. W. Gerdes, T. Giannantonio, D. A. Goldstein, R. A. Gruendl, J. Gschwend, G. Gutierrez, D. J. James, T. Jeltema, M. W. G. Johnson, M. D. Johnson, S. Kent, K. Kuehn, S. Kuhlmann, N. Kuropatkin, T. S. Li, M. Lima, H. Lin, M. A. G. Maia, M. March, J. L. Marshall, P. Martini, P. Melchior, F. Menanteau, R. Miquel, J. J. Mohr, E. Neilsen, R. C. Nichol, B. Nord, D. Petravick, A. A. Plazas, A. K. Romer, A. Roodman, M. Sako, E. Sanchez, V. Scarpine, R. Schindler, M. Schubnell, M. Smith, R. C. Smith, M. Soares-Santos, F. Sobreira, E. Suchyta, M. E. C. Swanson, G. Tarle, D. Thomas, D. L. Tucker, V. Vikram, A. R. Walker, J. Weller and Y. Zhang, ArXiv e-prints (August 2017) arXiv:1708.01538.

27. Planck Collaboration, N. Aghanim, Y. Akrami, M. Ashdown, J. Aumont, C. Baccigalupi, M. Ballardini, A. J. Banday, R. B. Barreiro, N. Bartolo, S. Basak, R. Battye, K. Benabed, J.-P. Bernard, M. Bersanelli, P. Bielewicz, J. J. Bock, J. R. Bond, J. Borrill, F. R. Bouchet, F. Boulanger, M. Bucher, C. Burigana, R. C. Butler, E. Calabrese, J.-F. Cardoso, J. Carron, A. Challinor, H. C. Chiang, J. Chluba, L. P. L. Colombo, C. Combet, D. Contreras, B. P. Crill, F. Cuttaia, P. de Bernardis, G. de Zotti, J. Delabrouille, J.-M. Delouis, E. Di Valentino, J. M. Diego, O. Doré, M. Douspis, A. Ducout, X. Dupac, S. Dusini, G. Efstathiou, F. Elsner, T. A. Enßlin, H. K. Eriksen, Y. Fantaye, M. Farhang, J. Fergusson, R. Fernandez-Cobos, F. Finelli, F. Forastieri, M. Frailis, E. Franceschi, A. Frolov, S. Galeotta, S. Galli, K. Ganga, R. T. GénovaSantos, M. Gerbino, T. Ghosh, J. González-Nuevo, K. M. Górski, S. Gratton, A. Gruppuso, J. E. Gudmundsson, J. Hamann, W. Handley, D. Herranz, E. Hivon, Z. Huang, A. H. Jaffe, W. C. Jones, A. Karakci, E. Keihänen, R. Keskitalo, K. Kiiveri, J. Kim, T. S. Kisner, L. Knox, N. Krachmalnicoff, M. Kunz, H. Kurki-Suonio, G. Lagache, J.-M. Lamarre, A. Lasenby, M. Lattanzi, C. R. Lawrence, M. Le Jeune, P. Lemos, J. Lesgourgues, F. Levrier, A. Lewis, M. Liguori, P. B. Lilje, M. Lilley, V. Lindholm, M. López-Caniego, P. M. Lubin, Y.-Z. Ma, J. F. Macías-Pérez, G. Maggio, D. Maino, N. Mandolesi, A. Mangilli, A. Marcos-Caballero, M. Maris, P. G. Martin, M. Martinelli, E. Martínez-González, S. Matarrese, N. Mauri, J. D. McEwen, P. R. Meinhold, A. Melchiorri, A. Mennella, M. Migliaccio, M. Millea, S. Mitra, M.-A. Miville-Deschênes, D. Molinari, L. Montier, G. Morgante, A. Moss, P. Natoli, H. U. Nørgaard-Nielsen, L. Pagano, D. Paoletti, B. Partridge, G. Patanchon, H. V. Peiris, F. Perrotta, V. Pettorino, F. Piacentini, L. Polastri, G. Polenta, J.-L. Puget, J. P. Rachen, M. Reinecke, M. Remazeilles, A. Renzi, G. Rocha, C. Rosset, G. Roudier, J. A. Rubiño-Martín, B. Ruiz-Granados, L. Salvati, M. Sandri, M. Savelainen, D. Scott, E. P. S. Shellard, C. Sirignano, G. Sirri, L. D. Spencer, R. Sunyaev, A.-S. Suur-Uski, J. A. Tauber, D. Tavagnacco, M. Tenti, L. Toffolatti, M. Tomasi, T. Trombetti, L. Valenziano, J. Valiviita, B. Van Tent, L. Vibert, P. Vielva, F. Villa, N. Vittorio, B. D. Wandelt, I. K. Wehus, M. White, S. D. M. White, A. Zacchei and A. Zonca, ArXiv e-prints (July 2018) arXiv:1807.06209.

28. P. Zhang, M. Liguori, R. Bean and S. Dodelson, Physical Review Letters 99 (October 2007) 141302, arXiv:0704.1932

29. R. Reyes, R. Mandelbaum, U. Seljak, T. Baldauf, J. E. Gunn, L. Lombriser and R. E. Smith, Nature 464 (March 2010) 256, arXiv:1003.2185 [astro-ph.C0].

30. A. Amon, C. Blake, C. Heymans, C. D. Leonard, M. Asgari, M. Bilicki, A. Choi, T. Erben, K. Glazebrook, J. Harnois-Deraps, H. Hildebrandt, H. Hoekstra, B. Joachimi, S. Joudaki, K. Kuijken, C. Lidman, D. Parkinson, E. A. Valentijn and C. Wolf, ArXiv e-prints (November 2017) arXiv:1711.10999 
31. C. D. Leonard, P. G. Ferreira and C. Heymans, jcap 12 (December 2015) 051, arXiv:1510.04287.

32. S. Singh, S. Alam, R. Mandelbaum, U. Seljak, S. Rodriguez-Torres and S. Ho, ArXiv e-prints (March 2018) arXiv:1803.08915.

33. G.-B. Zhao, H. Li, E. V. Linder, K. Koyama, D. J. Bacon and X. Zhang, Phys. Rev. D 85 (June 2012) 123546, arXiv:1109.1846 [astro-ph.CO]

34. A. Hojjati, G.-B. Zhao, L. Pogosian, A. Silvestri, R. Crittenden and K. Koyama, Phys. Rev. D 85 (February 2012) 043508, arXiv:1111.3960 [astro-ph.C0]

35. D. Kirk, I. Laszlo, S. Bridle and R. Bean, MNRAS 430 (March 2013) 197, arXiv:1109.4536

36. E. J. Ruiz and D. Huterer, Phys. Rev. D 91 (March 2015) 063009, arXiv:1410.5832

37. A. Hojjati, L. Pogosian and G.-B. Zhao, jcap 8 (August 2011) 005, arXiv:1106.4543 [astro-ph.CO].

38. J. N. Dossett, M. Ishak and J. Moldenhauer, Phys. Rev. D 84 (December 2011) 123001, arXiv:1109.4583

39. L. Pogosian and A. Silvestri, Phys. Rev. D 94 (November 2016) 104014, arXiv: 1606.05339

40. S. Peirone, K. Koyama, L. Pogosian, M. Raveri and A. Silvestri, Phys. Rev. D 97 (February 2018) 043519, arXiv: 1712.00444

41. E. Bellini, A. J. Cuesta, R. Jimenez and L. Verde, jcap 2 (February 2016) 053, arXiv:1509.07816

42. M. Zumalacárregui, E. Bellini, I. Sawicki, J. Lesgourgues and P. G. Ferreira, jcap 8 (August 2017) 019, arXiv:1605.06102

43. A. Spurio Mancini, R. Reischke, V. Pettorino, B. M. Scháefer and M. Zumalacárregui, ArXiv e-prints (January 2018) arXiv:1801.04251

44. W. A. Hellwing, K. Koyama, B. Bose and G.-B. Zhao, Phys. Rev. D 96 (Jul 2017) 023515.

45. A. Peel, V. Pettorino, C. Giocoli, J.-L. Starck and M. Baldi, ArXiv e-prints (May 2018) arXiv: 1805.05146

46. L. Lombriser, F. Simpson and A. Mead, Physical Review Letters 114 (June 2015) 251101, arXiv:1501.04961

47. J. Armijo, Y.-C. Cai, N. Padilla, B. Li and J. A. Peacock, MNRAS 478 (August 2018) 3627, arXiv: 1801.08975

48. C. Hernández-Aguayo, C. M. Baugh and B. Li, MNRAS 479 (October 2018) 4824, arXiv:1801.08880.

49. E. Semboloni, C. Heymans, L. van Waerbeke and P. Schneider, MNRAS 388 (August 2008) 991, arXiv:0802.3978

50. X. Liu, B. Li, G.-B. Zhao, M.-C. Chiu, W. Fang, C. Pan, Q. Wang, W. Du, S. Yuan, L. Fu and Z. Fan, Physical Review Letters 117 (July 2016) 051101, arXiv:1607.00184 